\title{
Solving a Truck Dispatching Scheduling Problem Using Branch-and-Cut
}

To appear in Operations Research.

\author{
Robert E. Bixby \\ Department of Computational and Applied Mathematics \\ Rice University, Houston, Texas, USA
}

Eva K. Lee

Department of Industrial Engineering and Operations Research

Columbia University, New York, New York, USA

1994 
Bixby \& Lee/Truck Dispatching Scheduling Using Branch-and-Cut

\begin{abstract}
A branch-and-cut IP solver is developed for a class of structured 0/1 integer programs arising from a truck dispatching scheduling problem. This problem involves a special class of knapsack equality constraints. Families of facets for the polytopes associated with individual knapsack constraints are identified. In addition, a notion of "conflict graph" is utilized to obtain an approximating node-packing polytope for the convex hull of all $0 / 1$ solutions. The branch-and-cut solver generates cuts based on both the knapsack equality constraints and the approximating node-packing polytope, and incorporates these cuts into a tree-search algorithm that uses problem reformulation and linear programming-based heuristics at each node in the search tree to assist in the solution process. Numerical experiments are performed on large-scale real instances supplied by Texaco Trading \& Transportation, Inc. The optimal schedules correspond to cost savings for the company and greater job satisfaction for drivers due to more balanced work schedules and income distribution.
\end{abstract}

Subject Classification: Transportation, vehicle routing: dispatching of trucks for an oil company. Programming, integer, algorithms, cutting plane/facet generation: branch-and-cut algorithm for a structured 0/1 problem. 
In this paper we present a branch-and-cut algorithm for a structured 0/1 integer programming problem referred to as the "truck dispatching scheduling (TDS) problem." The TDS problem is a vehicle routing and scheduling problem that arose from an oil company's desire to better utilize regional truck fleets and drivers. Many studies on different variations of vehicle routing and scheduling have appeared in the literature (Bodin 1990, Golden and Assad 1988). In most of these, the emphasis has been on developing fast heuristic algorithms to find good feasible solutions (Bodin 1990, p. 575). In contrast, the branch-and-cut algorithm developed herein finds optimal solutions.

A classical approach for solving integer programming problems is branch-and-bound. However, for large scale problems, such an approach often fails due to excessive memory usage. In 1983 Crowder, Johnson and Padberg incorporated preprocessing and cut generation prior to initiating the branch-and-bound procedure, resulting in remarkable solution times for some difficult instances (some which were once thought to be intractable). By the end of the 1980's, the term branch-and-cut was being used to describe methods that utilize cutting-plane techniques not only at the root node, but at nodes within the search tree itself. In these methods, cuts generated at a given node are valid globally across the entire search tree. The branch-and-cut approach has been responsible for solving important hard combinatorial optimization problems. In particular, large-scale instances of the traveling salesman problem and the airline crew scheduling problem have been solved to proven optimality in reasonably short amounts of time (Applegate, Bixby, Chvátal and Cook 1994, Hoffman and Padberg 1991, 1993, Padberg and Rinaldi 1989, 1991).

Certainly, an important aspect of a successful application of branch-and-cut is the ability to generate deep cuts in a computationally efficient way. This ability is often enhanced by knowledge of the facet structure of the convex hull of $0 / 1$ solutions, or of certain approximating polytopes to this convex hull. Thus, an important component of this research involved a study of the polyhedral structure of the TDS problem.

Polyhedral results pertaining to the TDS problem are summarized in Section 2. First, we consider approximating knapsack equality polytopes associated with individual constraints of the TDS model. These knapsack equality polytopes are of course intimately related to their inequality counterparts. We consider various families of facet defining inequalities for the associated knapsack inequality polytope and state conditions when these inequalities also define facets of the equality polytope.

We also consider an approximating polytope derived from the entire system of constraints. In particular, by allowing a relaxation of the equality constraints and defining 
the notion of conflict graph, a node packing polytope is obtained, from which clique and odd cycle inequalities are generated.

The branch-and-cut solver is described in Section 3. The individual components include a problem preprocessor, a heuristic, a cut generator, and an LP solver. These components have become the "standard" components for successful branch-and-cut solvers in recent years (e.g., see Hoffman and Padberg 1991, 1993, Padberg and Rinaldi 1989). However, the specific features one should include in each component and the best way of interfacing the components with one another is still largely experimental. With this in mind we designed the code so that individual features/components can be switched on and off, allowing great flexibility in testing the effectiveness of different solution strategies. Also note that most branch-and-cut solvers are problem specific and exploit the underlying problem structure whenever possible. In this same spirit our solver is specially tailored to solve the TDS problem; though of course, many of the techniques and much of the code are applicable to general $0 / 1$ problems.

Results of numerical experiments on fourteen large-scale real instances supplied by Texaco Trading \& Transportation are summarized in Section 4. The results indicate that in combination, the system's components yield an effective IP solver for this class of problems, and that cut generation plays a major role in reducing the time to establish optimality on some of the more difficult problem instances.

We now turn to Section 1, where we describe the TDS problem and its 0/1 integer programming formulation.

\section{Problem Description and Formulation}

An oil company has dispatchers in each of 17 regions in the United States. Each dispatcher is responsible for assigning itineraries to drivers each day. The set of itineraries selected must enable the pickup of crude products at designated locations for delivery to pipeline entry points. Each truck carries a tank of the same volume. When picking up product, the entire tank is filled, and this is called a load. Products are picked up and delivered as loads. Thus, each itinerary specifies an alternating sequence of pickup/delivery actions. When there is more than one load of crude product at a given pickup point, multiple pickups at that point result. Thus, associated with each itinerary are loads, pickup points, delivery points, deadhead miles (i.e., miles driven with an empty truck), and total hours.

Itineraries are generated for each driver or group of drivers (called a driver set) by an 
automatic process prior to the $0 / 1$ integer programming problem formulation. Drivers are grouped in the same driver set provided they have identical driver parameters, including home base location, hours available, starting time, breathing equipment certification, etc. The automatic process will generate a large number of possible itineraries for each driver set according to the parameters of the driver set, as well as the parameters associated with pickup points, delivery points, and Department of Transportation (DOT) regulations (e.g., open/close times for pickup and delivery points, and maximum on-duty-time allowed by DOT). The initial generation process may generate two or more itineraries that haul the same loads, but in a different order. In this case, preliminary elimination is done to select only the itinerary with the smallest cost. (The determination of the cost of an itinerary is discussed below.)

The primary objectives of the TDS problem are to minimize deadhead miles and equalize the loaded drive-time of the drivers. By minimizing deadhead miles, it is expected that trucks and drivers will be on the road fewer hours each day, thereby lowering fuel and maintenance costs, and improving safety for the drivers. Also, since driver payroll is based on the amount of time a driver is hauling product, by equalizing loaded drive-time, overall driver satisfaction should be increased. In order to reflect both objectives, to each generated itinerary a weight is assigned that incorporates the associated number of deadhead miles and a penalty associated with loaded drive-time being above or below a prespecified target level. The company anticipates that even a five percent reduction in deadhead miles could result in significant savings. In addition, obtaining optimal dispatches via solving the integer programs allows for more consistency in the dispatch creation in different locations, it lowers the training curve for dispatchers, and enables easier substitution and relocation of dispatchers.

There are two types of constraints in the $0 / 1$ integer programming formulation of the TDS problem. One type arises from the number of times each pickup point must be visited, and the second type arises from the number of drivers in each driver set. The parameters associated with the formulation are as follows:

$n=$ number of itineraries

$c_{j}=$ weight associated with the $j$ th itinerary

$p=$ number of pickup points

$b_{i}=$ number of times pickup point $i$ must be visited

$a_{i j}=$ number of times pickup point $i$ is visited according to itinerary $j$

$q=$ number of driver sets 


$$
\begin{aligned}
& g_{k}=\text { number of drivers in the } k \text { th driver set } \\
& n_{k}=\text { number of itineraries generated for the } k \text { th driver set. }
\end{aligned}
$$

In matrix form, the problem can be stated as

$$
\begin{aligned}
\operatorname{minimize} c^{T} x & & & \\
\text { subject to } A x & =b & & \text { (pickup point constraints) } \\
H x & =g & & \text { (driver set constraints) } \\
x & \in B^{n}, & &
\end{aligned}
$$

where $c=\left(c_{1}, \ldots, c_{n}\right)^{T} \in \Re^{n}, c>0 ; A=\left[a_{i j}\right]$ is a $p \times n$ nonnegative integral matrix; $b=\left(b_{1}, \ldots, b_{p}\right)^{T} \in Z^{p}, b>0 ; g=\left(g_{1}, \ldots, g_{q}\right)^{T} \in Z^{q}, g>0 ; B=\{0,1\} ;$ and $H$ is a $q \times n$ matrix of the form

$$
H=\left[\begin{array}{cccc}
1_{n_{1}}^{T} & & & \\
& 1_{n_{2}}^{T} & & \\
& & \ddots & \\
& & & 1_{n_{q}}^{T}
\end{array}\right],
$$

where $1_{n_{k}}$ is the vector in $\Re^{n_{k}}$ in which every entry is 1 , and $n_{1}+\cdots+n_{q}=n$. Furthermore, the $i$ th pickup point constraint $a_{i}^{T} x=b_{i}, 1 \leq i \leq p$, is of the form

$$
\sum_{j \in R_{i 1}} x_{j}+\sum_{j \in R_{i 2}} 2 x_{j}+\cdots+\sum_{j \in R_{i b_{i}}} b_{i} x_{j}=b_{i}
$$

where each $b_{i}$ is a small positive integer and $R_{i m}, 1 \leq m \leq b_{i}$, are disjoint subsets of $\{1, \ldots, n\}$.

Note that, by itself, a column of the matrix $A$ does not capture all the information about the associated itinerary. Indeed, it only reflects how many times each pickup point is to be visited. A separate database specifies detailed information about the itinerary, and once an optimal solution to the IP is obtained, the solution is then passed to a translation program to decipher. Once deciphered, estimated arrival and departure times for each location are compared across the dispatch to look for situations where too many drivers are scheduled to be at a given location at the same time. Adjustments are made as necessary and the final dispatch is generated. For the given test instances, we remark that no adjustments were actually needed; the optimal solution returned by the integer 
programming solver satisfied the time-space requirements.

\section{Polyhedral Theory for the TDS Problem}

\subsection{Preliminaries}

In this section we give an overview of the "cutting" aspect of a branch-and-cut approach for solving an integer programming problem.

Let $P^{I P}$ denote the convex hull of integer solutions of problem (1), let $P^{L P}$ denote the polytope associated with the linear programming relaxation, and let $\bar{x}$ be a solution of minimize $\left\{c^{T} x: x \in P^{L P}\right\}$. If $\bar{x}$ is integral, then we are done. If not, one attempts to generate one or more valid inequalities $\pi_{i}^{T} x \leq \pi_{i 0}$ for $P^{I P}$ such that $\pi_{i}^{T} \bar{x}>\pi_{i 0}$. One then adds these inequalities to the linear programming relaxation and obtains an updated solution $\bar{x}$ of the linear program minimize $\left\{c^{T} x: x \in P^{L P}, \pi_{i}^{T} x \leq \pi_{i 0}\right\}$. Again, if $\bar{x}$ is integral, we are done. If not, the process is repeated, saving only those valid inequalities generated from previous iterations that have been active (binding) at $\bar{x}$ within the last few iterations.

An effective way of generating valid inequalities that cut off the current fractional solution is to generate facet defining inequalities for various approximating polytopes of $P^{I P}$. In this work we make use of approximating knapsack equality polytopes associated with individual constraints of the TDS problem, and an approximating node packing polytope associated with a graph, called the conflict graph, obtained by considering all the constraints simultaneously.

\subsection{Facets of Approximating Polytopes}

Let $P^{i}$ denote the approximating polytope $P^{i}=\operatorname{conv}\left\{x \in B^{n}: a_{i}^{T} x=b_{i}\right\}$, where $a_{i}^{T} x=b_{i}$ is the $i$ th pick-up point constraint in the system $A x=b$. Since $a_{i}^{T} x=b_{i}$ is of the form (2), we need only to consider the general knapsack equality polytope

$$
S_{b}:=\operatorname{conv}\left\{x \in B^{n}: \sum_{j \in R_{1}} x_{j}+\sum_{j \in R_{2}} 2 x_{j}+\cdots+\sum_{j \in R_{b}} b x_{j}=b\right\}
$$

Clearly, the inequalities $x_{j} \geq 0$ and $x_{j} \leq 1$ determine all of the facets of $S_{1}$. Hence, the polyhedral structure of $S_{b}$ is only of interest when $b \geq 2$.

For simplicity of exposition, in what follows we assume that $\cup_{i=1}^{b} R_{i}=N$, where $N:=\{1, \ldots, n\}$. It is straightforward to extend the results to the general case. We 
also assume $\left|R_{1}\right| \geq b+1$, which is realistic for the TDS model since every pickup point constraint of the given collection of problem instances satisfies this condition. Together, these two assumptions imply that $S_{b}$ has dimension $n-1$.

In Theorem 1 we record $\sum_{k=1}^{b-1}\left(\begin{array}{c}\left|R_{1}\right| \\ k\end{array}\right)+\sum_{k=2}^{\lfloor b / 2\rfloor}\left|R_{k}\right|+1$ distinct facets of $S_{b}, b \geq 2$. For each facet we list one representative inequality from the family of valid inequalities defining it. These representative inequalities are in fact facet defining for the inequality counterpart of $S_{b}$ (that is, the full-dimensional polytope obtained by replacing the equality sign in (3) with less than or equal to), and could be obtained via lifting of certain minimal covers or $(1, k)$ configurations. Proofs that these inequalities are indeed facet defining for $S_{b}$ are given in Lee 1993a, 1993b and 1994. (Also, see Weismantel 1994 for related results.) What is important for the application at hand is that each representative inequality has most (in some cases, all) of the coefficients associated with variables with indices in $R_{1}$ equal to zero. For the problem instances motivating this work, a typical constraint has $\left|R_{1}\right|$ much greater than $\left|R_{i}\right|$ for $i=2, \ldots, b$; consequently, this representative inequality is the sparsest among all inequalities in the family. Since maintaining sparsity in the augmented constraint matrix is desirable when implementing a branch-and-cut algorithm, these representative inequalities are the ones actually used in the cut generation phase of the algorithm described in Section 3.

In the statement of the theorem we adopt the convention that a summation or union is vacuous if the lower limit is greater than the upper limit.

Theorem 1. Let $b \geq 2$, and let $S_{b}$ be defined as in (3), where $\cup_{i=1}^{b} R_{i}=N$ and $\left|R_{1}\right| \geq b+1$.

(a) Let $k \in\{1,2, \ldots, b-1\}$, let $T \subseteq R_{1}$ with $|T|=k$, and assume $\cup_{i=1}^{k-1} R_{b-i} \neq \emptyset$. Then the inequality

$$
\sum_{j \in T} x_{j}+\sum_{i=b-k+1}^{b} \sum_{j \in R_{i}}(k-b+i) x_{j} \leq k
$$

defines a facet of $S_{b}$.

(b) Let $i_{0} \in\{1, \ldots,\lfloor b / 2\rfloor\}$, and let $j_{0} \in R_{i_{0}}$. Then the inequality

$$
x_{j_{0}}+\sum_{i=b-i_{0}+1}^{b} \sum_{j \in R_{i}} x_{j} \leq 1
$$

defines a facet of $S_{b}$.

(c) Assume $\cup_{i=\lfloor b / 2\rfloor+1}^{b-1} R_{i} \neq \emptyset$, let $i_{0}=\min \left\{i \in\{\lfloor b / 2\rfloor+1, \ldots, b-1\}: R_{i} \neq \emptyset\right\}$, and 
suppose $\cup_{i=b-i_{0}+1}^{\lfloor b / 2\rfloor} R_{i}=\emptyset$. Then the inequality

$$
\sum_{i=i_{0}}^{b} \sum_{j \in R_{i}} x_{j} \leq 1
$$

defines a facet of $S_{b}$.

Note that if $j_{0} \in R_{1}$, then the associated facet identified in part (b) is identical to the facet associated with the set $T=\left\{j_{0}\right\}$ identified in part (a).

We next consider an approximating polytope derived by considering all the constraints simultaneously. For convenience of notation, let $\mathcal{A}=\left(\begin{array}{l}A \\ H\end{array}\right)$ and $\hat{b}=\left(\begin{array}{l}b \\ g\end{array}\right)$, where $A, H, b$ and $g$ are defined as in Section 1. Then the TDS polytope $P^{I P}$ can be expressed as $P^{I P}=\operatorname{conv}\left\{x \in B^{n}: \mathcal{A} x=\hat{b}\right\}$. Now consider the relaxation $P \leq=\operatorname{conv}\left\{x \in B^{n}:\right.$ $\mathcal{A} x \leqq \hat{b}\} \quad$ of $P^{I P}$. Although several classes of valid / facet defining inequalities for $P \leq$ are known (Nemhauser and Wolsey 1988, p. 237; Ferreira, Martin and Weismantel 1993), the polyhedral structure is less well-understood than that of a single knapsack inequality polytope (Nemhauser and Wolsey 1988, p. 290). One important class of valid inequalities for $P \leq$ is the minimal cover inequalities: $\sum_{i \in C} x_{i} \leq|C|-1$, where $C \subseteq N$ is a minimal set satisfying $\sum_{i \in C} a^{i} \not \hat{b}$, where $a^{i}$ denotes the $i$ th column of $\mathcal{A}$. Since for the given TDS problem instances no pickup point requires more than three visits per day, the components of the right-hand-side vector $b$ are either 1,2 or 3 . Consequently, one can readily find many minimal covers $C$ of cardinality 2 , and moreover, minimal covers of cardinality 3 or greater occur infrequently.

With this in mind we now define the conflict graph which allows us to approximate $P \leq$ by a node packing polytope. This idea extends that suggested by Johnson and Nemhauser (1991) who proposed obtaining clique inequalities based on a single knapsack constraint. It can also be viewed as a natural extension of the intersection graph associated with a pure $0 / 1$ integer matrix.

Definition 1. The conflict graph associated with the system $\mathcal{A} x \leqq \hat{b}, x \in B^{n}$ is the graph $G_{C}=(N, E)$, where

$$
\begin{aligned}
& N=\{1,2, \ldots, n\} \quad \text { and } \\
& E=\left\{j k: j, k \in N, j \neq k, \text { and } a^{j}+a^{k} \not \equiv \hat{b}\right\},
\end{aligned}
$$

where $a^{j}$ and $a^{k}$ denote distinct columns in $\mathcal{A}$.

Clearly, if $j k \in E$, then $x_{j}+x_{k} \leq 1$ is a valid inequality for $P^{I P}$. Hence, if $A_{C}$ denotes 
the edge-node incidence matrix of $G_{C}$, then the node packing polytope

$$
P^{C}=\operatorname{conv}\left\{x \in B^{n}: A_{C} x \leqq 1\right\}
$$

satisfies $P \leq \subseteq P^{C}$. Consequently, $P^{I P} \subseteq P^{C}$, and one can generate valid inequalities for $P^{I P}$ by applying results from the theory of node packing polytopes (Chvátal 1975, Padberg 1973, Trotter 1975, Wolsey 1976b). The following example illustrates that cuts generated via the conflict graph are, in general, tighter than cuts obtained using only one constraint at a time:

Example 1. Let $P^{I P}=\operatorname{conv}\left\{x \in B^{5}: A x=b\right\}$, where

$$
A=\left[\begin{array}{lllll}
1 & 1 & 1 & 0 & 0 \\
0 & 1 & 1 & 0 & 1 \\
1 & 1 & 0 & 2 & 2
\end{array}\right] \text { and } b=\left[\begin{array}{l}
1 \\
1 \\
2
\end{array}\right]
$$

The conflict graph yields the clique inequalities: $x_{1}+x_{2}+x_{3}+x_{5} \leq 1$ and $x_{1}+x_{2}+x_{4}+x_{5} \leq$ 1 which are violated by the fractional solution $\bar{x}=[1 / 2,1 / 2,0,0,1 / 2]^{T}$. However, no inequality generated from a single row of the system $A x=b$ is violated by this solution.

\section{The Branch-and-Cut IP Solver}

\subsection{Overview}

Recent implementations of branch-and-cut algorithms (e.g., see Hoffman and Padberg 1991, 1993, Padberg and Rinaldi 1991) have indicated that in addition to cut generation and clever branching strategies, other computational devices, such as preprocessing and heuristics, also play an important role in the solution process. However, the details of a "good" implementation — including exactly which techniques to incorporate, how to implement these techniques, and how the components of a branch-and-cut solver should interface with one another - are still largely experimental. Moreover, depending on the application in mind certain techniques in a given implementation may be tailored for the specific structure of the problem, while other techniques are of a general nature and could be effective on a wide variety of problems.

The Branch-and-Cut IP Solver described herein is designed to solve to optimality the real instances of the TDS problem, and consequently, it exploits the TDS problem structure when it is advantageous to do so. On the other hand, many of the techniques 
are applicable more generally; some being extensions of techniques described in Hoffman and Padberg (1993), and some being based on the polyhedral results in Section 2 .

The first task of the system is to preprocess the user-supplied linear programming formulation. Preprocessing reformulates and tightens the constraint matrix by removing redundant rows and columns, and fixing variables if possible. The preprocessing techniques are used throughout the entire branch-and-cut tree to reduce the size of subsequent linear programs and eliminate redundancy among rows and columns.

After initial preprocessing, we solve the linear program relaxation and obtain a lower bound for the original problem. Next, the heuristic is called. If the heuristic succeeds, we then perform reduced-cost fixing using the reduced-costs from the linear programming relaxation, the lower bound, and the upper bound. If enough variables are fixed, we eliminate columns that are fixed to zero and one, and preprocess the problem again. If the heuristic fails, we either branch or proceed to the constraint generator as described below.

Once the reduced-cost fixing is done, the linear program is solved again. If the solution is not integral, the constraint generator is called. The particular polyhedral cuts that we generate are knapsack cuts (as described in Section 2.2), clique inequalities and odd cycle inequalities. The latter two types of cuts are generated over a partial conflict graph. They are then tightened by lifting to include other nodes in the graph.

The cuts obtained, which are valid across the entire tree, are then appended to the constraint matrix, and the linear program is solved again. We iterate through this process until one of the following occurs: (i) an integral solution is obtained, (ii) the linear program is infeasible, (iii) no more cuts are generated, (iv) the number of cuts generated exceeds a prespecified value, or (v) the objective value of the linear programs have not improved much. If (i) occurs, the node is fathomed. The next node is chosen based on the bestestimate strategy; i.e., we select the node with the smallest LP value among all active nodes. (Note that the current LP value of a node serves only as an estimate since the true value may be different due to the addition of cuts). However, we move directly to this next node only if the integral objective value is greater than that of the current integral upper bound. Otherwise, we update the integral upper bound, return to the root node, resolve the linear program with the current cuts appended, and perform reduced-cost fixing. Only after all this is completed do we move to the node in the tree with the smallest LP value; at which point we reconstruct this node by resolving the associated linear program, again with the current cuts appended and the current variables fixed.

If (ii) occurs, the node is fathomed, and we continue our search with the remaining 
active nodes. If (iii), (iv) or (v) occur, we perform the heuristic again. If the heuristic succeeds and produces a better upper bound, reduced-cost fixing is performed at the root. After this, or if the heuristic does not produce a better upper bound, we select a variable to branch on and create two new nodes. We experimented with branching on the smallest indexed fractional variable, and branching on the most infeasible fractional variable. The former selection worked better in the overall performance on the real instances, although in a few cases, using the most infeasible fractional variable was superior (see Section 4). Condition (iv) is used in our system as one of the criteria to move on since the gap between the linear program relaxation and the optimal integral solution in several of the test problems is very small (see Table I). As a result, it is not always easy to detect the "tailing off" of the procedure as in (v). We remark that a small gap does not imply that the integer programs are easy to solve. For example, problem p6000 in MIPLIB (Bixby et al, 1993) has a relative gap of only $0.007 \%$, and yet it is very difficult to solve, as evidenced in Hoffman and Padberg 1991.

A flow chart of the entire system is given in Figure 1.

\subsection{Preprocessing and Reformulation}

Preprocessing techniques fix variables permanently, remove redundant rows, and check for inconsistencies among the constraints (Crowder, Johnson and Padberg 1983, Hoffman and Padberg 1991, 1993, Savelsbergh 1994, Suhl and Szymanski 1994). Some of the features in our preprocessor are frequently mentioned in the literature of branch-and-cut, in particular the removal of duplicate, multiple and redundant rows. In addition, we extend the row inclusion and the clique fixing techniques for set partitioning problems (Hoffman and Padberg 1993) to our integral matrix, and incorporate conflict fixing based on the idea from Section 2. A brief description of these techniques is given below.

Row Inclusion/Multiple-Inclusion Fixing. This procedure searches for rows that are properly contained in a multiple of another row; i.e., an appropriate multiple of one row is an "inclusion" of the other. To be precise, let $J$ and $K$ be the sets of column indices for the nonzero entries of two distinct rows, say rows 1 and 2, respectively, and suppose $J \subseteq K$. Thus, we have the constraints

$$
\begin{aligned}
\sum_{j \in J} a_{1 j} x_{j} & =b_{1} \\
\sum_{j \in J} a_{2 j} x_{j}+\sum_{j \in K \backslash J} a_{2 j} x_{j} & =b_{2} .
\end{aligned}
$$


Furthermore, suppose $a_{i j} \geq 0$ for all $i$ and $j$, and suppose $a_{1 j} \leq\left(b_{1} / b_{2}\right) a_{2 j}$ for all $j \in J$. Then $x_{k}=0$ for all $k \in(K \backslash J) \cup\left\{j \in J: a_{1 j}<\left(b_{1} / b_{2}\right) a_{2 j}\right\}$. In this case, row 2 can be removed and row 1 can be updated.

In the implementation, we first distinguish between constraints for pickup points and driver sets. Corresponding to each pickup point constraint, we record the number of distinct driver sets appearing in the row, and the total number of itineraries for each driver set. For those rows with itineraries from exactly one driver set, we perform the inclusion test for this row and the corresponding driver set constraint.

Next we do a pairwise comparison of pickup rows. Assume the $q$ driver sets are labeled $1, \ldots, q$. If the labels appearing in one row of a pair also appear in the other, we check that the number of itineraries in each represented driver set of the former is less than or equal to that of the latter. If this is the case, we perform the inclusion test, checking along the smaller of the associated index sets. For example, assume only driver sets 2 and 3 are represented in row 1 and only driver sets 1,2,3 and 4 are represented in row 2. Let $m_{i j}$ denote the number of nonzero coefficients associated with driver set $j$ in row $i$. If $m_{12} \leq m_{22}$ and $m_{13} \leq m_{23}$, then the inclusion test would be performed, with the candidate set $J$ consisting of the $m_{12}+m_{13}$ indices in row 1 .

Creating a Clique Matrix. Let $G_{C}$ be the conflict graph associated with the integral matrix $\mathcal{A}:=\left(\begin{array}{l}A \\ H\end{array}\right)$ and the vector $\hat{b}=\left(\begin{array}{l}b \\ g\end{array}\right)$. Choose a row $i$ with right-hand-side 1 , and let $M_{i}=\left\{j \in N: a_{i j}=1\right\}$. If there exists a complete subgraph $K$ of $G_{C}$ such that $M_{i} \subseteq K$, then since $\sum_{j \in K} x_{j} \leq 1$ and $\sum_{j \in M_{i}} x_{j}=1$, it follows that $x_{j}=0$ for all $j \in K \backslash M_{i}$. To implement this, we select a node $v \in \operatorname{adj}\left(M_{i}\right)$, the set of nodes in $N \backslash M_{i}$ that are adjacent to at least one node in $M_{i}$. If degree $(v)<\left|M_{i}\right|$, discard $v$. Otherwise, scan through the adjacency list of $v$. If it does not contain all the nodes in $M_{i}$, then discard $v$. Otherwise, $v$ is part of a complete subgraph containing $M_{i}$, so we set $x_{v}=0$. Repeat the above process for each distinct $v \in \operatorname{adj}\left(M_{i}\right)$.

Subsequent Conflict-Fixing of Variables. If some variable $x_{k}$ has been fixed to one,

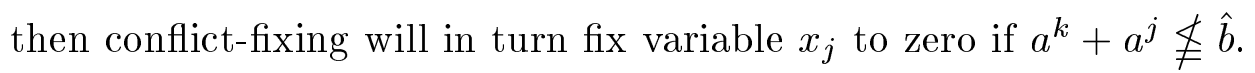

\subsection{The Heuristic}

The heuristic provides a means of obtaining integer feasible solutions quickly, and is used repeatedly within the branch-and-cut search tree. A good heuristic - one that produces 
good integer feasible solutions - is a crucial component in the branch-and-cut algorithm since it provides an upper bound for reduced-cost fixing at the root, and thus allows reduction in the size of the linear program that must be solved. This in turn may reduce the time required to solve subsequent linear programs at nodes within the search tree. In addition, a good upper bound also enables us to fathom more active nodes, which is extremely important in solving large-scale integer programs as they tend to create many active nodes leading to memory explosion.

Our heuristic works by sequentially fixing variables and solving the corresponding linear programs. It incorporates logical and conflict fixing based on the TDS problem structure. Fine tuning and selection of various parameters within the heuristic were based on extensive numerical experiments using the given TDS problem instances. The heuristic algorithm terminates when either an integer feasible solution is obtained, or the linear program is infeasible. If it returns an integer feasible solution with a lower objective value than the upper bound of the integer program, the upper bound is updated and we move once again to the reduced-cost fixing routine. Otherwise, we turn to cut generation.

At a node of the branch-and-bound tree, the heuristic first solves the LP-relaxation associated with that node. If a fractional solution is obtained and an upper bound for the integer program exists, the heuristic then performs reduced-cost fixing to fix nonbasic variables at their corresponding lower bound and upper bound. In the latter case, for the rows covered by these variables, implied fixing is performed, and the appropriate rows are removed. (A row is said to be covered by the variables set equal to one if the sum of the associated coefficients equals the right-hand-side.) For other rows containing these variables but not covered by them, we perform conflict fixing of the variables. After removing all variables fixed to zero, the linear program is solved again. If there are still fractional values in the optimal solution, variables (both basic and nonbasic) at value one will be set to one. Then, for specified parameters $\alpha, \beta$, and $t o l$, the heuristic computes $x_{\text {max }}=\max \left\{x_{j}:\right.$ tol $\left.<x_{j}<1-t o l\right\}$, and $x_{\text {min }}=\min \left\{x_{j}:\right.$ tol $\left.<x_{j}<1-t o l\right\}$, and sets $x_{j}=1$ for $x_{j} \geq x_{m a x}-\alpha * t o l$, and $x_{j}=0$ for $x_{j} \leq x_{\min }+\beta * t o l$. After extensive numerical tests, we set $t o l=0.001, \alpha=20$ and $\beta=15$. Special care is taken to ensure that no variable is set to one, and then subsequently set to zero when $x_{\max }-\alpha *$ tol $\leq x_{j} \leq x_{\min }+\beta *$ tol. In addition, when all the fractional variables fall outside the range $t o l<x_{j}<1-t o l$, the code automatically resets $x_{\max }$ to $1-t o l$, and $x_{\min }$ to $t o l$. After these additional variables are fixed, the LP solver is called again, and the process continues.

We experimented with the frequency of heuristic calls on our test problems, and found 
that applying the heuristic at every node of the search tree is ineffective in improving the solution time. Indeed, on average, 20 linear programs are solved per heuristic call, and the solution time for each of these linear programs ranges from 0.2 to 1.9 seconds. As a result, applying the heuristic at each node tends to be computationally expensive. Based on our experiments, the present code is set to call the heuristic as follows: at the root node, the heuristic is called before the first cut generation and also before branching begins; and within the branch-and-bound tree, it is invoked whenever the node is at a depth of a multiple of 8 from the root.

\subsection{Continuous Reduced Cost Implications}

Reduced cost fixing is a well-known and important idea in the literature of integer programming. In a pure zero-one linear programming minimization problem, given the continuous optimal objective value $z_{l p}$, the continuous optimal reduced cost $d_{j}$, and a true upper bound, $z^{+}$, on the zero-one optimal objective function value, then for all nonbasic variables $x_{j}$ in the continuous optimal solution, the following are true:

(a) If $x_{j}=0$ in the continuous solution and $z^{+}-z_{l p}<d_{j}$, then $x_{j}=0$ in every zero-one solution.

(b) If $x_{j}=1$ in the continuous solution and $z^{+}-z_{l p}<-d_{j}$, then $x_{j}=1$ in every zero-one solution.

In our branch-and-cut implementation, before branching, global reduced cost fixing (that is, permanently fixing variables at their respective upper and lower bounds) is performed at the root node whenever an improved integral upper bound is obtained, or whenever the objective value of the linear programming relaxation at the root node improves (due to the addition of cuts). Local fixing is performed at each node of the search tree using the local LP value and the current best upper bound. On those nodes where the heuristic is called, local fixing is performed both before and after the heuristic call.

\subsection{Constraint Generation}

Let $\bar{x}$ be the fractional solution obtained at a given node in the tree, and let $L=\{j \in$ $\left.N: \bar{x}_{j}=0\right\}, \quad F=\left\{j \in N: 0<\bar{x}_{j}<1\right\} \quad$ and $\quad U=\left\{j \in N: \bar{x}_{j}=1\right\}$. Since we would like to identify a valid inequality, $\pi^{T} x \leq \pi_{0}$, which is violated by $\bar{x}$, we can ignore the set $L$ in the identification phase. However, unlike the set packing problem, due to the non-0/1 entries in the constraint matrix and right-hand-side of the TDS problem, it is advantageous 
not to ignore the variables indexed by $U$ when generating valid inequalities based on the conflict graph. More specifically, for the set packing problem, since every neighbor $k$ of a node in $U$ in the intersection graph necessarily satisfies $\bar{x}_{k}=0$, one cannot obtain violated valid inequalities by focusing on graph structures (e.g., cliques and odd cycles) involving nodes in $U$ (Hoffman and Padberg 1993, p. 675). The same is not necessarily true when considering the conflict graph associated with a problem with non 0/1 data. For example, consider again Example 1 from Section 2.2. The conflict graph yields the valid inequality $x_{1}+x_{2}+x_{4}+x_{5} \leq 1$, which is violated by the fractional solution $\bar{x}=[0,1,0,1 / 2,0]^{T}$.

Let us now focus on generating valid inequalities for the approximating polytope $P^{C}$ defined in Section 2.2. Let $G_{F \cup U}=\left(F \cup U, E_{F \cup U}\right)$ denote the subgraph of $G_{C}$ induced by the node set $F \cup U$. Since the graph $G_{F \cup U}$ can be quite large, rather than computing the entire graph, in our implementation only subgraphs of $G_{F \cup U}$ are generated. The size of the generated subgraph is controlled by adjusting the tolerance used to check for nonzero values of the solution vector $\bar{x}$. Our current tolerance is set to 0.001. Since one can think of the set $F$ as being controlled by adjusting the tolerance, we will continue to use the notation $G_{F \cup U}$ to denote these subgraphs.

Clique Generation. We generate violated clique inequalities of the subgraph $G_{F \cup U}$ using the following two procedures. Note that we preserve the graph $G_{F \cup U}$ and only use a copy when implementing each procedure.

(a) Select a vertex $s$ with maximum degree and use it to initialize a "clique set" $K$. Throw away nodes not adjacent to $s$ and update the adjacency lists of the remaining nodes. Continue to add nodes to $K$ as follows. If $v$ is the last vertex added to $K$, find a vertex $u$ in the adjacency list of $v$ that has maximum degree and is not in $K$, add it to $K$, and throw away all nodes that are not in the adjacency list of $u$, again updating the adjacency lists of the remaining nodes. If no such $u$ exists, stop; a clique has been found, in which case we check to see if the associated clique inequality is violated by the current fractional solution.

This procedure is repeated on the subgraph of $G_{F \cup U}$ obtained by deleting the starting node $s$ and its associated clique. We continue to repeat - each time deleting all previous starting nodes and associated clique nodes - until either $10 \%$ of the nodes in $F \cup U$ have been selected as starting nodes, or until the deletion process eliminates 
all of $F \cup U$.

(b) First, for each row $i$, define the index set(s)

$$
\begin{aligned}
M_{i} & =\left\{j \in F \cup U: a_{i j}=1\right\}, & & \text { if the right hand side of row } i \text { equals } 1 \\
M_{i j_{0}} & =\left\{j \in F \cup U: a_{i j}=b\right\} \cup\left\{j_{0}\right\}, & & \text { if the right hand side of row } i \text { equals } b,
\end{aligned}
$$

where $b \geq 2$, and $j_{0} \in F \cup U, a_{i j_{0}} \in\{1,2, \ldots, b-1\}$. Clearly, $M_{i}$ and $M_{i j_{0}}$ are the node sets of complete subgraphs of $G_{F \cup U}$. Enlarge such a subgraph by finding the set $K \subseteq(F \cup U) \backslash M_{i}\left(K \subseteq(F \cup U) \backslash M_{i j_{0}}\right)$ such that each node in $K$ is adjacent to every node in $M_{i}\left(M_{i j_{0}}\right)$. Then apply procedure (a) to identify one or several cliques in the subgraph induced by $K$. In the implementation, for each $i$, if the number of sets $M_{i j_{0}}$ is less than or equal to 32 we perform this procedure for each such set. Otherwise, we perform it for either $20 \%$ of them or 32 of them (whichever is greater), where $j_{0}$ is chosen in decreasing order of the values of $\bar{x}_{j_{0}}$. (We remark that in the numerical experiments on the given problem instances, with our chosen tolerance, we never generated a partial conflict graph with more than 200 vertices. In other applications, it may be necessary to place an upper bound on the number of sets $M_{i j_{0}}$ processed.) For each clique that is identified, we check to see if the associated inequality is violated. If no violated inequality is found, we form a new set $M_{i}^{\prime}\left(M_{i j_{0}}^{\prime}\right)$ consisting of half the indices in $M_{i}\left(M_{i j_{0}}\right)$ selected in decreasing order of $\bar{x}_{j}$. We then repeat the above procedure, still restricting $K \subseteq(F \cup U) \backslash M_{i}\left(K \subseteq(F \cup U) \backslash M_{i j_{0}}\right)$.

Chordless Odd Cycles. It is well-known that if $K$ is an odd cycle without chords, then the odd cycle inequality $\sum_{u \in K} x_{u} \leq(|K|-1) / 2$ is valid for $P^{C}$ and defines a facet of the node packing polytope associated with the restriction of $P^{C}$ to the node set $K$. An effective heuristic for obtaining an odd cycle $K$ involves performing breadth-first search on a chosen node $s$. An odd cycle is obtained whenever a cross edge is encountered between two nodes, say $u$ and $v$, on the same level of the breadth-first tree (Cormen, Leiserson and Rivest 1990). To recover the cycle, trace back along the paths to these two nodes until arriving at a common ancestor, say $w$ (which could be different from $s$ ). Let $P_{u}$ and $P_{v}$ denote the node sets along the paths from $w$ to $u$, and from $w$ to $v$, respectively excluding $w$ in both cases. Clearly $P_{u} \cup P_{v} \cup\{w\}$ forms the node set of an odd cycle. To determine if the odd cycle is chordless, we first consider successive nodes in $P_{u} \backslash\{u\}$. If a node is found whose adjacency list contains a node in $P_{v}$, stop; the cycle has a chord. If, on the other hand, the adjacency list of every node in $P_{u} \backslash\{u\}$ is disjoint from $P_{v}$, we 
then determine if node $u$ is adjacent to a node in $P_{v} \backslash\{v\}$. If so, we again conclude that the cycle has a chord; if not, the cycle is chordless. (Note that the breadth first search strategy precludes the existence of a chord involving the common ancestor $w$.) Once a chordless odd cycle is found, the associated inequality is checked to see if it is violated by the current fractional solution. If not, we perform lifting of exactly 10 nodes (chosen in decreasing order of the associated variable values) from the remaining nodes and check if the final inequality is violated.

This entire procedure is repeated using different starting root nodes — first selecting $10 \%$ of the nodes in $F \cup U$ in decreasing order of the associated variable values, and then selecting $10 \%$ from the remaining nodes using a pseudo-random selection process.

Facets for Individual Knapsack Equalities. This procedure identifies facets for individual knapsack polytopes of the form $S_{b}$ using the facet defining inequalities obtained in Section 2.2. In the current implementation, this procedure is specifically tailored to solve the given TDS problem instances. Since in these instances the largest coefficient in any knapsack equality constraint is 3 , the current system is set to only search for facet defining inequalities associated with $S_{2}$ and $S_{3}$, namely

$$
\begin{aligned}
x_{j_{0}}+\sum_{j \in R_{2}} x_{j} \quad \leq 1, \quad j_{0} \in R_{1} \\
x_{j_{0}}+\sum_{j \in R_{3}} x_{j} \leq 1, \quad j_{0} \in R_{1} \\
\sum_{j \in T} x_{j}+\sum_{j \in R_{2}} x_{j}+\sum_{j \in R_{3}} 2 x_{j} \leq 2, \quad T \subseteq R_{1},|T|=2 \\
\sum_{j \in R_{2}} x_{j}+\sum_{j \in R_{3}} x_{j} \leq 1,
\end{aligned}
$$

where (7) is associated with $S_{2}$ and (8) - (10) are associated with $S_{3}$.

Whether or not the inequalities generated by these methods yield deep cuts and/or high dimensional faces of $P^{I P}$ depends in large part on how closely the polytopes $P^{i}$ and $P^{C}$ approximate $P^{I P}$. Nevertheless, one can tighten the generated inequalities by lifting.

The Lifting Procedure. Let $\pi^{T} x \leq \pi_{0}$ be a valid inequality for $P^{I P}$, and let $J=$ $\left\{j \in N: \pi_{j} \neq 0\right\}$. If $y \in \Re^{n}$, let $y_{J}$ denote the vector in $\Re^{|J|}$ with the components $y_{i}, i \in N \backslash J$, removed. Similarly, let $\mathcal{A}_{J}$ denote the $(p+q) \times|J|$ matrix obtained by removing the columns indexed by $i \in N \backslash J$ from the constraint matrix $\mathcal{A}$. For an index $k \in N \backslash J$, we determine a lifting coefficient $\hat{\pi}_{k}$ for $x_{k}$ by first solving the lifting problem: 
$z_{k}=\operatorname{maximize}\left\{\pi_{J}^{T} x_{J}: \mathcal{A}_{J} x_{J} \leqq \hat{b}-a^{k}, x_{j} \in\{0,1\}, j \in J\right\}$, where $a^{k}$ denotes the $k$ th column of $\mathcal{A}$. Then $\hat{\pi}_{k}=\pi_{0}-z_{k}$. If it is positive, we update $\pi_{k} \leftarrow \hat{\pi}_{k}$ and $J \leftarrow J \cup\{k\}$, and repeat the process. Note that the order in which the variables are lifted is important, in the sense that different lifting orders can potentially produce different valid inequalities (Padberg 1973).

We estimate the lifting coefficients by solving the linear programming relaxation of the lifting problem. Another approach, which avoids solving a linear program, extends the idea described in Hoffman and Padberg (1993) for the set packing problem. The method is based on the approximation: $\max \left\{\pi_{J}^{T} x_{J}: A_{J} x_{J} \leqq \hat{b}-a^{k}, x_{j} \in\{0,1\}\right.$ for all $\left.j \in J\right\} \leq$ $\min \left\{u^{T}\left(\hat{b}-a^{k}\right): u^{T} A_{J} \geqq \pi_{J}^{T}, u \geqq 0, u\right.$ integral $\}$. In our implementation, we restrict the components of $u$ to be binary, and estimate its optimal objective value by a greedy algorithm.

In general, the more variables that are lifted, the tighter the final cut. However, lifting can be computationally expensive, since the lifting problem itself is a $0 / 1$ integer program. Hence, some sort of compromise must be made regarding the number of variables to be lifted. Our current procedure performs lifting until a maximum of 100 positive lifting coefficients are obtained.

\section{Numerical Tests and Results}

The preprocessor, the reduced-cost fixing routine, the heuristic and the constraint generator are integrated into a Branch-and-Cut IP Solver using CPLEX 2.1 as the intermediate LP Solver. The code is written in C, and not counting the LP solver and the comment statements, has about 16,000 lines. Numerical tests on 14 real instances supplied by Texaco Trading \& Transportation, Inc. were run on a SUN super SPARCstation 10 model 41 with super cache.

Table I provides a description of the problem instances. Cols, Rows, and Nonzeros denote, respectively, the number of columns, the number of rows, and the number of non-zeros in the constraint matrix; $Z_{L P}$ and $Z_{I P}$ denote the optimal objective values for the initial linear programming relaxation and the integer program; and Gap denotes the difference between $Z_{L P}$ and $Z_{I P}$. A value of zero for the gap does not imply that the LP solver returned an integral feasible solution from the linear programming relaxation. We remark that the company was able to solve to optimality some smaller instances with 2000 or fewer variables in an acceptable time, but was not able to do so on larger instances. 
Table II summarizes the results using our plain branch-and-bound (i.e., with the preprocessor, reduced-cost routine, heuristic, and constraint generator switched off) and using CPLEXMIP 2.1. BB Nodes and BB time denote the number of nodes in the terminal search tree and the time required to solve to optimality using our plain branch-and-bound, and CPLEX Nodes and CPLEX Time denote corresponding information when CPLEXMIP is used. Our branch-and-bound code solves all the instances and outperforms CPLEXMIP on 6 of them. CPLEXMIP solves 12 of the 14 problem instances, and has better running times on 8 of them. Our code uses best-node strategy for node selection, and is set to branch on the fractional binary variable with smallest index. For CPLEXMIP we used the default parameters - best-node, and automatic variable selection (using either pseudo reduced-costs or maximum infeasibility). We note that for the $z s^{*}$ problems, solution times are much better with our code (by as much as ten-fold compared to what is reported here) if we instead set it to branch on the most infeasible fractional binary variable. However, this strategy increases the running times for the five $z b^{*}$ problems. For uniform reporting of the computational results, in the tables we only report numerical results for our chosen default settings - for which all problems can be solved in reasonable time.

Table III provides a comprehensive picture of the branch-and-cut system and the effort expended by each of its major components. Prepr. Calls, R-cost Calls and Heur. Calls denote the number of times the preprocessor, the reduced-cost routine, and the heuristic were called, and Prepr. Time, R-cost Time and Heur. Time denote their respective times. Heur. Success indicates the number of times the heuristic returned an integral solution, and Cuts indicates the number of cuts generated. The time spent managing cut generation is listed under Cuts Time, and reflects the cumulative CPU time of all calls to the cut generator, including the time required to create the partial conflict graphs, the time to generate and add cuts to the database of the LP solver, and the time to delete cuts that have been inactive for four consecutive LP solves. Finally, BC Nodes and Total CPU secs. denote the number of nodes in the branch-and-cut tree and the total time of the entire branch-and-cut process.

Table IV compares the results in Tables II and III, measured in terms of the ratios of the number of nodes and the total CPU time for the branch-and-cut algorithm relative to those for plain branch-and-bound and CPLEXMIP. Observe that branch-and-cut outperformed branch-and-bound on all but one test problem (zb1all.1). Moreover, for those problems for which branch-and-bound required more than 10,000 seconds (i.e., zb2all, zb2all.1, zm3all, zs2short, zs3short) branch-and-cut substantially reduced the CPU time 
required to solve them to optimality. In all these cases, the time is reduced by more than $88 \%$. The overall average time reduction for branch-and-cut over branch-and-bound for the 14 instances is $77.6 \%$. Compared to CPLEXMIP, we again see that branch-and-cut yielded better times in all but one instance (zs3short). For those problems for which CPLEXMIP required more than 10,000 seconds (i.e., zb2all, zb2all.1, zm3all, zs2short) solution times are reduced by more than $77 \%$. The overall average time reduction for branch-and-cut over CPLEXMIP for all 14 instances is $74.3 \%$.

Table V records the performance of the heuristic routine when it is used as a "standalone" procedure. $Z_{H E U R}$ denotes the first heuristic value obtained, and Heur. Time denotes the time to compute this value. Note that the first call to the heuristic returned an optimal value for seven of the fourteen problem instances (four of which had a gap of zero). Moreover, for two "hard" problem instances (zb2all and zb2all.1) the heuristic returned an optimal solution within 30 seconds. Thus, for these two cases, the bulk of the total solution time recorded in Table III (3702.5 and 1190.4 seconds, respectively) was spent closing the gap between the lower and upper bounds in order to establish optimality.

To illustrate the importance of cut generation in our branch-and-cut solver, Table VI reports the results when only the preprocessing, reduced-cost and heuristic routines are switched on, and compares these with the results from Table III. For those problems with total CPU time over 10,000 seconds when running with cuts disabled (i.e., zb2all, zb2all.1, zm3all and zs2short) the time reduction achieved by switching on the cut generator is more than $72 \%$. For the 10 problems for which cuts were generated, the average reduction in running time is $68.4 \%$. To further emphasize the key role that cut generation plays, consider zb2all and zb2all.1. In each case, the first call to the heuristic returned an optimal solution in less than 30 seconds. Nevertheless, without cut generation, it took 13,501.7 and 14,231.9 seconds, respectively, to solve them to optimality. By invoking the cut generator the times were reduced to 3702.5 and 1190.4 seconds (reductions of $72.6 \%$ and $91.6 \%$ ) respectively. We remark that although CPLEXMIP also found the optimal solutions for these two instances relatively quickly (after solving 14 and 22 branch-and-bound nodes, respectively), it failed to solve them to proven optimality, and strenuous effort $(205,700$ and 149,500 nodes in the respective search trees) was expended to close the gaps by only 1.2163 and 1.1420 , respectively.

We experimented with ways to handle nonactive cuts efficiently. When a cutpool was employed, less than $3 \%$ of the cuts were reused. We estimated the CPU time for cut generation with and without a cutpool, and the former required over $20 \%$ in excess of the 
latter. Hence, our branch-and-cut solver defaults to cut generation without a cutpool; and cuts are removed after being inactive for four consecutive LP solves.

We also measured the relative frequencies of the three classes of generated cuts on each of the fourteen instances. Cuts derived via the knapsack equalities accounted for $10 \%$ - $15.3 \%$ of the overall number of cuts; clique inequalities accounted for $59.5 \%-81.2 \%$; and odd cycle inequalities accounted for $5.2 \%-23.7 \%$. Disabling the knapsack cuts increased the computational time by $17.2 \%$ on average over all instances, and by $45.2 \%$ on the four most difficult instances (zb2all, zb2all.1, zm3all and zs2short). The resulting increases from disabling the clique and odd cycle cut generation routines were $54.3 \%$ and $20.1 \%$, respectively, over all the instances. Also, we remark that the conflict graph proved to be crucial in generating clique and odd cycle inequalities. Over $99 \%$ of the violated cliques and odd cycles generated in the code could not be constructed using only a single constraint at a time.

As with any branch-and-cut system, the performance of the LP solver — which is repeatedly called to solve sequences of linear programs - is crucial to the overall performance of the branch-and-cut system. Therefore, we experimented with various features of CPLEX to determine which features could be exploited to speed up the overall process of the system. The system is now set to use primal reduced-cost pricing to solve the initial linear programming relaxation, and steepest-edge primal pricing for successive resolves of the linear programs within the heuristic and after reduced-cost fixing in the branch-andcut tree. Finally, steepest-edge dual pricing is used for solving the linear programs during the resolve phase in the cutting plane routine and within the branching phase.

In summary, the numerical tests indicate that the branch-and-cut system significantly reduces the total CPU time to solve to optimality some hard instances of the TDS problem. Table III clearly demonstrates the effectiveness of the combination of the system's components. Moreover, using the reported times in Tables II and VI one can calculate that the combination of the preprocessor, the reduced-cost routine and the heuristic alone yields an average time reduction of $61.1 \%$ over plain branch-and-bound. However, Table VI and the performance of CPLEXMIP on the $z b 2^{*}$ instances give strong evidence that cut generation plays a leading role in improving the solution time on the most difficult 
instances.

\section{Acknowledgement}

We would like to thank Donna McDougald Peacock of Texaco, Inc. for taking the time to explain the truck dispatching system and obtaining test problems for us, and Texaco Trading \& Transportation, Inc. for allowing the test problems to be released. Also, we would like to thank Karla Hoffman for sharing her experience in implementing branchand-cut systems. Finally, we would like to thank two anonymous referees for their helpful comments on an earlier version of this paper. This work was completed while the second author was at Rice University. We acknowledge support through AFOSR Grant F4962092-J-0053.

\section{References}

Applegate D., R. Bixby, V. Chvátal and W. Cook. 1994. Solving Traveling Salesman Problems. Preprint.

Balas, E. AND E. Zemel. 1984. Lifting and Complementing Yields all the Facets of Positive Zero-one Programming Polytopes. In: R.W. Cottle et al., eds., Mathematical Programming, Proceedings of the International Conference on Mathematical Programming. $13-24$.

Bixby, R.E., E.A. Boyd, S.S. Dadmehr, And R.R. Indovina. 1993. The MIPLIB Mixed Integer Programming Library. COAL Bulletin 22.

Bodin, L.D. 1990. Twenty Years of Routing and Scheduling. Operations Research 38, $571-579$.

Chvátal, V. 1975. On Certain Polytopes Associated with Graphs. Journal of Combinatorial Theory B13, 138-154.

Cormen, T.H., C.E. Leiserson, And R.L. Rivest. 1990. Introduction to Algorithms. MIT Press, Cambridge, MA.

CPLEX MAnuAL. 1993. Using the CPLEX $X^{T M}$ Callable Library and the CPLEX $X^{T M}$ Mixed Integer Library. CPLEX Optimization, Inc., Incline Village, Nevada.

Crowder, H., E.L. Johnson, And M. Padberg. 1983. Solving Large-scale Zeroone Linear Programming Problems. Operations Research 31, 803-834.

Ferreira, C.E., A. Martin and R. Weismantel. 1993. Facets for the Multiple 
Knapsack Problem. Preprint. Konrad-Zuse-Zentrum für Informationstechnik Berlin.

Golden, B.L. And A. Assad, Editors. 1988. Vehicle Routing: Methods and Studies (Studies in Management Science and Systems, Volume 16). North Holland, Amsterdam.

Gomory, R.E. 1969. Some Polyhedra Related to Combinatorial Problems. Linear Algebra and Its Applications 2, 451-558.

Grötschel, M. And O. Holland. 1991. Solution of Large-scale Symmetric Travelling Salesman Problems. Mathematical Programming 51, 141-202.

Hammer, P.L. And U.N. Peled. 1982. Computing Low-capacity 0-1 Knapsack Polytopes.

Zeitschrift für Operations Research 26, 243-249.

Hoffman, K.L. AND M. PADBERG. 1991. Improving LP-representations of Zero-one Linear Programs for Branch-and-cut. ORSA Journal on Computing 3, 121-134.

Hoffman, K.L. And M. Padberg. 1993. Solving Airline Crew-Scheduling Problems by Branch-and-cut. Management Science 39, 657-682.

Johnson, E.L., M.M. Kostreva, And U.H. Suhl. 1985. Solving 0-1 Integer Programming Problems Arising from Large-scale Planning Models. Operations Research 33, 803-819.

Johnson, E.L. And G.L. Nemhauser. 1991. Recent Developments and Future Directions in Mathematical Programming. Research Report. Computational Optimization Center, Georgia Institute of Technology, Atlanta, Georgia.

LeE, E.K. 1993a. Solving Structured 0/1 Integer Programming Problems Arising from Truck Dispatching Scheduling Problems. Ph.D. Thesis. Rice University, Houston, Texas.

LeE, E.K. 1993b. Facets of Special Knapsack Equality Polytopes. Technical Report TR93-38. Rice University, Houston, Texas.

LeE, E.K. 1994. On Facets of Knapsack Equality Polytopes. To appear in Journal of Optimization Theory and Applications 94, No. 1, 223-239.

Nemhauser, G.L. And L.A. Wolsey. 1988. Integer and Combinatorial Optimization. Wiley, New York.

PAdberg, M. 1973. On the Facial Structure of Set Packing Polyhedra. Mathematical Programming 5, 199-215.

Padberg, M. And G. Rinaldi. 1989. A Branch-and-cut Approach to a Traveling 
Salesman Problem with Side Constraints. Management Science 35, 1393-1412.

Padberg, M. And G. Rinaldi. 1991. A Branch-and-cut Algorithm for the Resolution of Large-scale Symmetric Traveling Salesman Problems. SIAM Review 33, 60-100.

Savelsbergh M.W.P. 1994. Preprocessing and Probing for Mixed Integer Programming Problems. ORSA Journal on Computing 6, 445-454.

Suhl, U.H. And R. Szymanski. 1994. Supernode processing of mixed integer models. Computational Optimization and Applications 3, 317-331.

Trotter, L.E. 1975. A Class of Facet-producing Graphs for Vertex Packing Polyhedra. Discrete Mathematics 12, 373-388.

Weismantel, R. 1994. On the 0/1 Knapsack Polytope. Research Report SC 94-1. Konrad-Zuse-Zentrum für Informationstechnik Berlin.

Wolsey, L.A. 1976a. Facets and Strong Valid Inequalities for Integer Programs. Operations Research 24, 367-372.

Wolsey, L.A. 1976b. Further Facet Generating Procedures for Vertex Packing Polytopes. Mathematical Programming 11, 158-163.

Zemel, E. 1978. Lifting the Facets of Zero-one Polytopes. Mathematical Program$\operatorname{ming} 15,268-277$. 
Figure 1

\section{Branch-and-Cut Flowchart ${ }^{a}$}

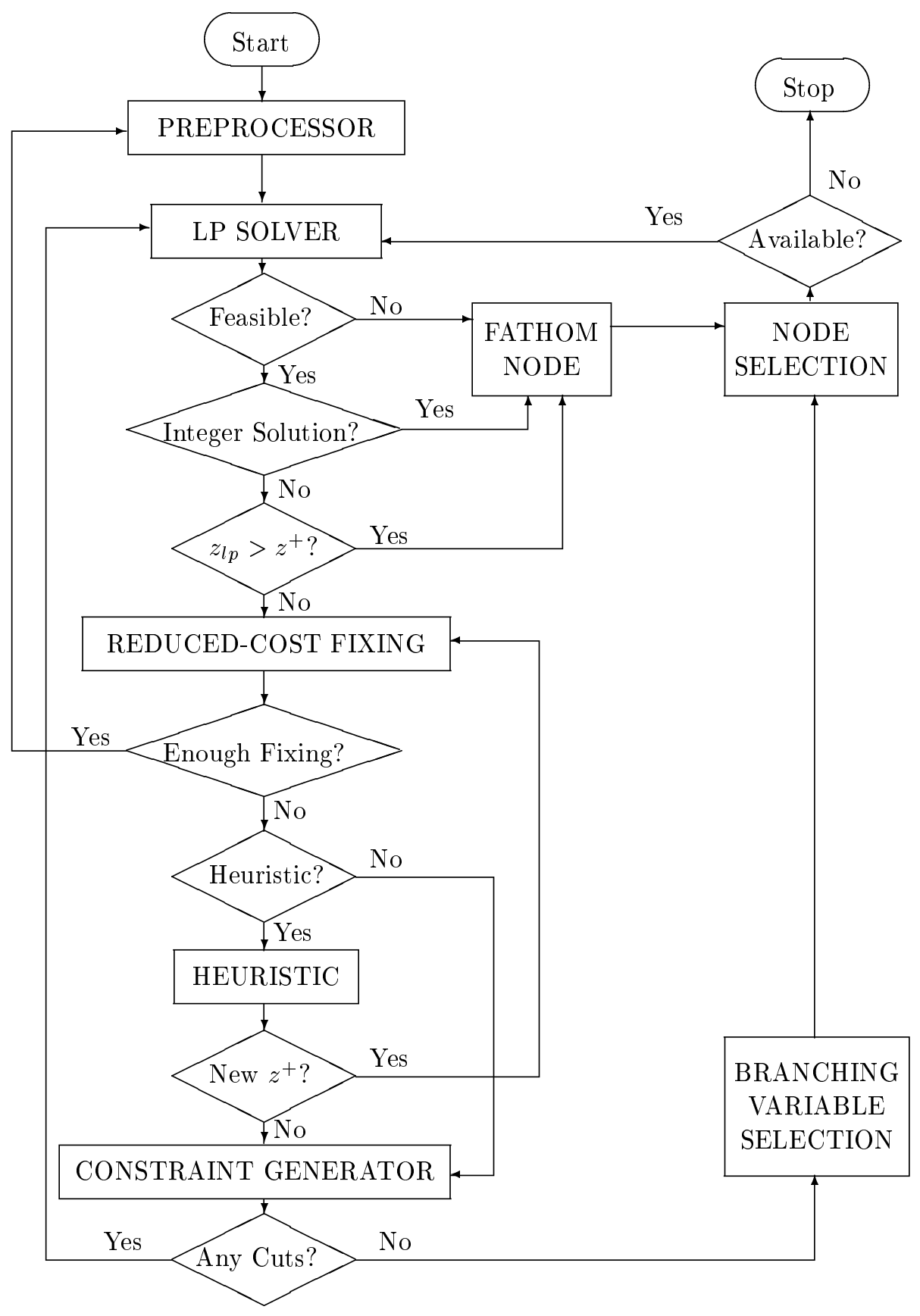

${ }^{a} z_{l p}=$ LP objective value; $z^{+}=$objective value of best integer solution 
Table I

\begin{tabular}{|lrrrrrr|}
\hline \multicolumn{7}{|c|}{ Problem Characteristics } \\
\hline Problem & Cols. & Rows & Nonzeros & \multicolumn{1}{c|}{$Z_{L P}$} & \multicolumn{1}{c|}{$Z_{I P}$} & Gap \\
\hline zb1all & 27768 & 47 & 114138 & 2319.80 & 2319.80 & 0.00 \\
zb1all.1 & 24771 & 47 & 103020 & 2012.28 & 2012.28 & 0.00 \\
zb2all & 24172 & 49 & 98607 & 2630.37 & 2639.40 & 9.03 \\
zb2all.1 & 21801 & 51 & 89670 & 2259.50 & 2268.00 & 8.50 \\
zb3all & 5712 & 37 & 22411 & 1456.00 & 1456.00 & 0.00 \\
zm1all & 5301 & 26 & 22236 & 1444.60 & 1449.00 & 4.40 \\
zm2all & 11706 & 28 & 54216 & 1284.95 & 1285.29 & 0.34 \\
zm2new & 3636 & 29 & 18190 & 1419.69 & 1426.00 & 6.31 \\
zm3all & 10849 & 30 & 44916 & 1305.24 & 1319.26 & 14.02 \\
zs1new & 2089 & 28 & 8403 & 1527.73 & 1539.60 & 11.87 \\
zs1short & 115544 & 26 & 666323 & 889.00 & 889.00 & 0.00 \\
zs2short & 26306 & 27 & 138322 & 904.71 & 919.21 & 14.50 \\
zs3short & 11273 & 25 & 59509 & 1048.70 & 1071.25 & 22.55 \\
zs3area & 4030 & 34 & 17154 & 1918.59 & 1924.00 & 5.41 \\
\hline
\end{tabular}


Table II

\begin{tabular}{|lrrrr|}
\hline \multicolumn{5}{c|}{ Numerical Results Using } \\
\hline \multicolumn{5}{c|}{ Plain Branch-and-Bound and CPLEXMIP } \\
\hline Problem & BB Nodes & BB Time & CPLEX Nodes & CPLEX Time \\
\hline zb1all & 65 & 172.5 & 17 & 92.0 \\
zb1all.1 & 35 & 88.7 & 46 & 260.9 \\
zb2all & 62644 & 340690.0 & $205700^{a}$ & $426600.1^{a}$ \\
zb2all.1 & 55460 & 333047.0 & $149500^{b}$ & $459775.8^{b}$ \\
zb3all & 7 & 2.7 & 13 & 9.3 \\
zm1all & 214 & 76.7 & 155 & 49.6 \\
zm2all & 36 & 16.9 & 28 & 45.6 \\
zm2new & 992 & 135.5 & 847 & 209.2 \\
zm3all & 43990 & 156158.0 & 37505 & 25317.9 \\
zs1new & 14446 & 2842.7 & 1682 & 243.9 \\
zs1short & 12 & 171.8 & 3 & 97.8 \\
zs2short & 16298 & 168292.0 & 10594 & 25288.6 \\
zs3short & 8578 & 26874.8 & 2805 & 562.3 \\
zs3area & 4632 & 1357.2 & 1979 & 2138.0 \\
\hline
\end{tabular}

${ }^{a}$ Not proven optimal (out of memory). Best upper bound 2639.4, best lower bound 2631.5863

${ }^{b}$ Not proven optimal (out of memory). Best upper bound 2268, best lower bound 2260.6420. 
Table III

\begin{tabular}{|c|c|c|c|c|c|c|c|c|c|c|c|}
\hline \multicolumn{12}{|c|}{$\begin{array}{c}\text { Numerical Results for } \\
\text { Branch-and-Cut }\end{array}$} \\
\hline Problem & $\begin{array}{l}\text { Prepr. } \\
\text { Calls }\end{array}$ & $\begin{array}{l}\text { Prepr. } \\
\text { Time }\end{array}$ & $\begin{array}{c}R \text {-cost } \\
\text { Calls }\end{array}$ & $\begin{array}{l}R \text {-cost } \\
\text { Time }\end{array}$ & $\begin{array}{l}\text { Heur } \\
\text { Calls }\end{array}$ & $\begin{array}{c}\text { Heur. } \\
\text { Success }\end{array}$ & $\begin{array}{l}\text { Heur. } \\
\text { Time }\end{array}$ & Cuts & $\begin{array}{l}\text { Cuts } \\
\text { Time }\end{array}$ & $\begin{array}{c}B C \\
\text { Nodes }\end{array}$ & $\begin{array}{c}\text { Total } \\
\text { CPU secs }\end{array}$ \\
\hline zb1all & 1 & 0.2 & 0 & 0 & 1 & 1 & 9.9 & $0^{a}$ & 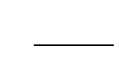 & 0 & 11.0 \\
\hline zb1all.1 & 2 & 0.1 & 0 & 0 & 1 & 1 & 120.2 & $0^{a}$ & $\bar{F}$ & 0 & 120.3 \\
\hline zb2all & 2 & 0.1 & 12 & 2.1 & 1 & 1 & 26.0 & 456 & 2017.3 & 0 & 3702.5 \\
\hline zb2all.1 & 3 & 0.6 & 8 & 0.9 & 1 & 1 & 29.9 & 405 & 732.5 & 0 & 1190.4 \\
\hline zb3all & 2 & 0.1 & 0 & 0 & 1 & 1 & 1.7 & $0^{a}$ & 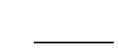 & 0 & 2.1 \\
\hline zm1all & 3 & 0.1 & 19 & 3.5 & 3 & 2 & 1.7 & 442 & 3.7 & 66 & 14.9 \\
\hline zm2all & 3 & 0.1 & 1 & 0.1 & 1 & 1 & 0.2 & 5 & $0.0^{b}$ & 0 & 0.4 \\
\hline zm2new & 3 & 0.2 & 16 & 1.9 & 3 & 3 & 7.2 & 821 & 22.3 & 26 & 45.4 \\
\hline zm3all & 1 & 0.1 & 11 & 2.5 & 5 & 2 & 7.1 & 5413 & 3529.1 & 198 & 5624.3 \\
\hline zs1new & 3 & 0.1 & 1 & 0.1 & 1 & 1 & 6.9 & 59 & 12.3 & 0 & 19.6 \\
\hline zs1short & 1 & 0.0 & 0 & 0 & 1 & 1 & 24.2 & $0^{a}$ & _ & 0 & 24.2 \\
\hline zs2short & 3 & 0.6 & 27 & 6.5 & 3 & 3 & 9.7 & 3091 & 2764.1 & 149 & 4882.7 \\
\hline zs3short & 3 & 0.5 & 63 & 20.5 & 3 & 3 & 4.8 & 4902 & 1503.9 & 156 & 3026.8 \\
\hline zs3area & 3 & 0.1 & 6 & 0.8 & 2 & 2 & 10.9 & 453 & 37.2 & 36 & 54.6 \\
\hline
\end{tabular}

${ }^{a}$ Gap is zero and optimal IP Solution obtained after first call to heuristic.

${ }^{b}$ Time is less than 0.05 seconds. 
Table IV

\begin{tabular}{|c|c|c|c|c|}
\hline \multicolumn{5}{|c|}{ Branch-and-Bound and CPLEX vs. Branch-and-Cut } \\
\hline Problem & $\frac{B C \text { Nodes }}{B B \text { Nodes }}$ & $\frac{B C \text { Time }}{B B \text { Time }}$ & $\frac{B C \text { Nodes }}{C P L E X \text { Nodes }}$ & $\frac{B C \text { Time }}{\text { CPLEX Time }}$ \\
\hline zb1all & 0 & 0.064 & 0 & 0.120 \\
\hline zb1all.1 & 0 & 1.356 & 0 & 0.461 \\
\hline zb2all & 0 & 0.011 & 0 & $\leq 0.009$ \\
\hline zb2all.1 & 0 & 0.004 & 0 & $\leq 0.003$ \\
\hline zb3all & 0 & 0.778 & 0 & 0.226 \\
\hline zm1all & 0.308 & 0.194 & 0.426 & 0.300 \\
\hline zm2all & 0 & 0.024 & 0 & 0.009 \\
\hline zm2new & 0.026 & 0.335 & 0.031 & 0.217 \\
\hline zm3all & 0.005 & 0.036 & 0.005 & 0.222 \\
\hline zs1new & 0 & 0.007 & 0 & 0.080 \\
\hline zs1short & 0 & 0.141 & 0 & 0.247 \\
\hline zs2short & 0.009 & 0.029 & 0.014 & 0.193 \\
\hline zs3short & 0.018 & 0.113 & 0.056 & 1.416 \\
\hline zs3area & 0.008 & 0.040 & 0.018 & 0.097 \\
\hline
\end{tabular}


Table V

\begin{tabular}{|c|c|c|c|c|c|}
\hline \multicolumn{6}{|c|}{ Objective Values from First Call to Heuristic } \\
\hline Problem & $Z_{L P}$ & $Z_{I P}$ & $Z_{H E U R}$ & $Z_{H E U R}-Z_{I P}$ & $\begin{array}{l}\text { Heur. } \\
\text { Time }\end{array}$ \\
\hline zb1all & 2319.80 & 2319.80 & 2319.80 & 0 & 9.9 \\
\hline zb1all.1 & 2012.28 & 2012.28 & 2012.28 & 0 & 120.2 \\
\hline zb2all & 2630.37 & 2639.40 & 2639.40 & 0 & 26.0 \\
\hline zb2all.1 & 2259.50 & 2268.00 & 2268.00 & 0 & 29.9 \\
\hline zb3all & 1456.00 & 1456.00 & 1456.00 & 0 & 1.7 \\
\hline zm1all & 1444.60 & 1449.00 & 1452.00 & 3 & 0.7 \\
\hline zm2all & 1284.95 & 1285.29 & 1285.29 & 0 & 0.2 \\
\hline zm2new & 1419.69 & 1426.00 & 1428.70 & 2.70 & 4.6 \\
\hline zm3all & 1305.24 & 1319.26 & fail & - & 1.2 \\
\hline zs1new & 1527.73 & 1539.60 & 1543.20 & 3.60 & 6.9 \\
\hline zs1short & 889.00 & 889.00 & 889.00 & 0 & 24.2 \\
\hline zs2short & 904.71 & 919.21 & 922.21 & 3 & 3.2 \\
\hline zs3short & 1048.70 & 1071.25 & 1084.33 & 13.08 & 1.0 \\
\hline zs3area & 1918.59 & 1924.00 & 1976.50 & 52.50 & 4.9 \\
\hline
\end{tabular}


Table VI

\begin{tabular}{|c|c|c|c|c|c|c|c|c|c|c|c|}
\hline \multicolumn{12}{|c|}{$\begin{array}{c}\text { Numerical Results for Branch-and-Cut } \\
\text { With Cut Generator Switched Off }\end{array}$} \\
\hline Problem & $\begin{array}{r}\text { Prepr. } \\
\text { Calls }\end{array}$ & $\begin{array}{l}\text { Prepr. } \\
\text { Time }\end{array}$ & $\begin{array}{c}R \text {-cost } \\
\text { Calls }\end{array}$ & $\begin{array}{l}R \text {-cost } \\
\text { Time }\end{array}$ & $\begin{array}{r}\text { Heur. } \\
\text { Calls }\end{array}$ & $\begin{array}{l}\text { Heur. } \\
\text { Success }\end{array}$ & $\begin{array}{l}\text { Heur. } \\
\text { Time }\end{array}$ & $\begin{array}{l}\text { Total } \\
\text { Nodes }\end{array}$ & $\begin{array}{c}\text { Total } \\
\text { CPU secs }\end{array}$ & $\frac{B C-\text { Nodes }}{\text { Nodes }}$ & $\frac{B C-\text { Time }}{\text { Time }}$ \\
\hline zb1all & 1 & 0.2 & 0 & 0 & 1 & 1 & 9.9 & 0 & 11.0 & - & 1.000 \\
\hline zb1all.1 & 2 & 0.1 & 0 & 0 & 1 & 1 & 120.2 & 0 & 120.3 & - & 1.000 \\
\hline zb2all & 3 & 0.2 & 112 & 42.1 & 141 & 87 & 2009.2 & 30001 & 13501.7 & 0.000 & 0.274 \\
\hline zb2all.1 & 4 & 0.7 & 81 & 30.1 & 99 & 76 & 1601.2 & 27029 & 14231.9 & 0.000 & 0.084 \\
\hline zb3all & 2 & 0.1 & 0 & 0 & 1 & 1 & 1.7 & 0 & 2.1 & - & 1.000 \\
\hline zm1all & 4 & 0.2 & 20 & 3.7 & 4 & 3 & 2.7 & 162 & 58.9 & 0.407 & 0.253 \\
\hline zm2all & 3 & 0.2 & 1 & 0.1 & 1 & 1 & 0.2 & 2 & 0.5 & 0.000 & 0.800 \\
\hline zm2new & 3 & 0.2 & 18 & 2.1 & 5 & 4 & 6.3 & 96 & 47.3 & 0.271 & 0.960 \\
\hline zm3all & 3 & 0.2 & 242 & 71.2 & 59 & 21 & 137.1 & 27001 & 78201.3 & 0.007 & 0.072 \\
\hline zs1new & 4 & 0.3 & 132 & 41.2 & 7 & 3 & 23.1 & 8921 & 1309.1 & 0.000 & 0.015 \\
\hline zs1short & 1 & 0.0 & 0 & 0 & 1 & 1 & 24.2 & 0 & 24.2 & - & 1.000 \\
\hline zs2short & 3 & 0.6 & 187 & 71.3 & 91 & 62 & 192.2 & 15902 & 49821.3 & 0.009 & 0.098 \\
\hline zs3short & 3 & 0.5 & 92 & 29.1 & 89 & 70 & 190.8 & 7083 & 6012.7 & 0.022 & 0.503 \\
\hline zs3area & 4 & 0.2 & 59 & 19.2 & 13 & 10 & 5.3 & 3012 & 529.8 & 0.012 & 0.103 \\
\hline
\end{tabular}

